\title{
Exploration of the Galilean Moons using Electrodynamic Tethers for Propellantless Maneuvers and Self-Powering
}

\author{
Lorenzini E. C. ${ }^{1}$, Curreli D. ${ }^{2}$ and Zanutto D. ${ }^{2}$ \\ ${ }^{1}$ Departement of Mechanical Engineering, University of Padua, \\ e-mail: enrico.lorenzini@unipd.it \\ ${ }^{2}$ Centre of Studies and Activities for Space, CISAS - "G. Colombo", University of Padua, \\ e-mail: davide.curreli@unipd.it, denis.zanutto@studenti.unipd.it
}

\begin{abstract}
Recent studies have demonstrated the benefits of using electrodynamic tethers $(E D T)$ for the exploration of the inner region of the Jovian system. Intense planetary magnetic field and reasonable environmental plasma density make the electrodynamic interaction of the conductive tether with the plasmasphere strong. The interaction is responsible for a Lorentz force that can be conveniently used for propellantless maneuvers and extraction of electrical power for on board use. Jupiter and the four Galilean Moons represent an exceptional gravitational environment for the study of the orbital dynamics of an EDT. The dynamics of such a system was analyzed using a 3-body model, consisting of the planet plus one of its moons (Io in this work) and the EDT itself. New and interesting features appear, like for example the possibility to place the tether in equilibrium with respect to a frame co-rotating with the moon at points that do not coincide with the classical Lagrangian points for non-null electrodynamic forces.
\end{abstract}

Keywords. Jovian Environment, Plasma Torus, Space Plasma, Space Tethers, Electrodynamic Propulsion, Radiation Belts

\section{Introduction}

Jupiter's fast rotation and its strong magnetic field create a magnetosphere with unique features in the Solar System for its immense size. Jupiter's huge magnetosphere differs from most other planetary magnetospheres because it derives much of its plasma internally from the volcanic activity of the moon Io. The nature of Jovian environment (high magnetic field and moderate electron density in several regions) offers very attractive features for an EDT to operate. The motional electric field due to the relative motion of the $E D T$ with respect to the magnetic lines drives the collection of electrons from the plasmasphere onto the anodic portion of the conducting cable. As a consequence, an electric current flows along the tether driven by the potential difference between anode and cathode (where an hollow cathode emits electrons). The Lorentz force acting on the system due to the interaction of the current with the local magnetic field can be conveniently used for propellantless maneuvers within the Jovian system. Adopting an Orbital Motion Limited $(O M L)$ model the averaged current collected can be calculated using the $O M L$ formula (Sanmartin, 1993). When the electric current on the conductive wire is closed on a load, the EDT allows the extraction of electric power at the expense of the energy of the corotating plasma, which is thermodynamically cooled and slowed down. 


\section{Jupiter's Environment}

The Jovian magnetic field is very intense (4.2 Gauss at a Jovian radius) and its structure is complex because of the interactions with solar wind. Pioneer and Vojager missions and the radio observations provided some indications about the intensity of this magnetic field demonstrating that is inverted with respect to the Earth field, inclined and offset. Though in the literature fairly accurate models of magnetic field exist (for example the Octupole Model, named O4), the relatively simple Offset Tilted Dipole Model, named D4, is already a good approximation for the aims of this work.

Jupiter's magnetic field rotates at the same rotational speed of the planet, with a period of $9 \mathrm{~h} 55 \mathrm{~m}$, so the plasma entraped inside the magnetosphere corotates, with the planet, at very high velocities.

Tidal deformations inside Io produce extreme tectonics and volcanism: neutral gas continuously ejected by the moon is ionized and accelerated by the fast-flowing Jovian magnetosphere. Because of this activity, Io acts as a source of neutral material that is then ionized and taken to the state of a cold plasma, forming the plasma torus that surrounds Io's orbit.

The Divine-Garrett model gives a simplified description of the plasma environment by providing the densities of protons, electrons and six positive ion species as a function of the position vector. Plasma environment has a fundamental importance in the operations of the electrodynamic tether because the tether current is directly proportional to the plasma electron density.

\section{Perturbed Circular Restricted Three Body Problem}

The system formed by Jupiter, Io and EDT satellite represents an interesting example of three body problem. The attitude and orbital motion of the EDT satellite center of mass is governed by the classical circular restricted three body problem perturbed by the electrodynamic force $F_{e l}$ and its moments.

$$
\begin{aligned}
& \ddot{\vec{R}}+2 \vec{\Omega} \times \dot{\vec{R}}+\vec{\Omega} \times \vec{\Omega} \times \vec{R}=\vec{f}_{g r, 1}+\vec{f}_{g r, 2}+\vec{f}_{e l} \\
& {\left[\dot{I}_{c}\right] \vec{\omega}+\left[I_{c}\right] \dot{\vec{\omega}}+\vec{\omega} \times\left[I_{c}\right] \vec{\omega}=\vec{M}_{g r, 1}+\vec{M}_{g r, 2}+\vec{M}_{e l}}
\end{aligned}
$$

The tether is approximated by means of the Extended Dumbbell Model with two lumped masses at the tips of a rigid and massless cable of length L. The dynamics of the tether attitude angles, $\theta$ and $\varphi$, with respect to inertial frame, is as follows:

$$
\begin{aligned}
& \ddot{\theta}+2 \frac{\dot{L}}{L} \dot{\theta}-2 \dot{\theta} \dot{\varphi} \tan \varphi=\frac{1}{\cos \varphi}\left[\frac{3 \nu_{1}}{\rho_{1}^{5}}\left(\vec{\rho}_{1} \cdot \hat{u}\right)\left(\vec{\rho}_{1} \cdot \hat{v}\right)-\frac{3 \nu_{2}}{\rho_{2}^{5}}\left(\vec{\rho}_{2} \cdot \hat{u}\right)\left(\vec{\rho}_{2} \cdot \hat{v}\right)+\frac{M_{\theta, e l}}{I_{c}}\right] \\
& \ddot{\varphi}+2 \frac{\dot{L}}{L} \dot{\varphi}+\dot{\theta}^{2} \cos \varphi \sin \varphi=-\frac{3 \nu_{1}}{\rho_{1}^{5}}\left(\vec{\rho}_{1} \cdot \hat{u}\right)\left(\vec{\rho}_{1} \cdot \hat{w}\right)-\frac{3 \nu_{2}}{\rho_{2}^{5}}\left(\vec{\rho}_{2} \cdot \hat{u}\right)\left(\vec{\rho}_{2} \cdot \hat{w}\right)+\frac{M_{\varphi, e l}}{I_{c}}
\end{aligned}
$$

The orbital motion of the electrodynamic tethered satellite in the Io Torus was investigated and new triangular equilibrium positions were found which are displaced with respect to the classic Lagrangian points and rotated along the orbit (see Figure 1) by an angle in the direction opposite to the EDT force. The value of the rotation angle is a function of the electrodynamic interaction and, consequently, of size parameters like tether length, tether width and S/C mass. With the increase of tether length the superior point (near $L_{4}$ ) is stable and tends asymptotically to a location very close to Io, while the inferior point (near $L_{5}$ ) disappears gradually, losing its stable nature. These equilibrium positions of the perturbed system were obtained numerically by using an iterative algorithm. Small 


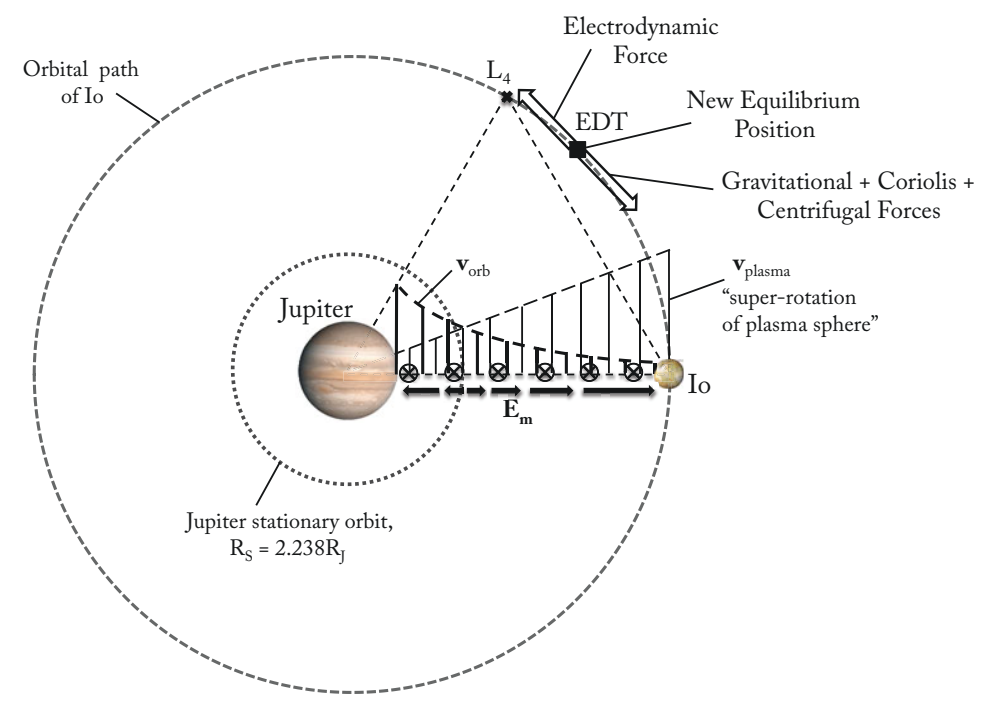

Figure 1. The EDT can stay in new equilibrium positions w.r.t. the synodic frame

motions around them were also studied with a linear variational analysis, by computing the eigenvalues of the linearized system. They consist of complex numbers with the same real part $R_{e}$ and frequencies $f_{1}$ and $f_{2}$.

$$
\begin{aligned}
& s_{1,2}=R_{e} \pm i f_{1} \\
& s_{3,4}=-R_{e} \pm i f_{2}
\end{aligned}
$$

Indeed, the magnetic field, electron density and relative velocity with respect to the plasma are variable along the orbit and consequently they modulate the electrodynamic force. The solutions of the characteristic polynomial are time and space-dependent: real parts of all eigenvalues change along the trajectory because of the modulation of $B, N_{e}$ and $v_{r e l}$. Three kinds of trajectories are possible as a function of the integral average of real parts of all eigenvalues: convergent, divergent and quasi-periodic orbits.

\subsection{Motion around the new equilibrium points}

The dynamics around the modified triangular Lagrangian points was investigated to identify mission profiles capable of exploring the inner zone of the Jovian system at the distance of Io that scans the whole Plasma Torus. The relevant trajectories exhibit large amplitude oscillations around the modified equilibrium positions. Several tether configurations of moderate sizes were studied to allow trajectories with useful levels of power extracted from the plasma environment. By adding an initial out-of-plane component the $\mathrm{S} / \mathrm{C}$ develops an harmonic oscillation in $z$-direction that lets the system explore and scan the Torus (see Figure 2).

\subsection{Rotating tether}

Rotating tethers are necessary to maintain the cable taut at all times by means of centrifugal forces, also when the natural gravity-gradient force is not able to exert a sufficient tension. It is possible in this way to keep the lateral bowing due to Lorentz force small. The Lorentz force acts as a lateral distributed load all along the cable.

The total tension is:

$$
T_{\text {tot }}=T_{g r}+T_{\text {spin }}=3 \omega_{\text {orb }}^{2} m_{1} L\left(\frac{m_{2}}{m_{1}+m_{2}}\right)+\omega_{\text {spin }} \frac{L}{2}\left(\frac{m_{1}+m_{2}}{2}\right)
$$




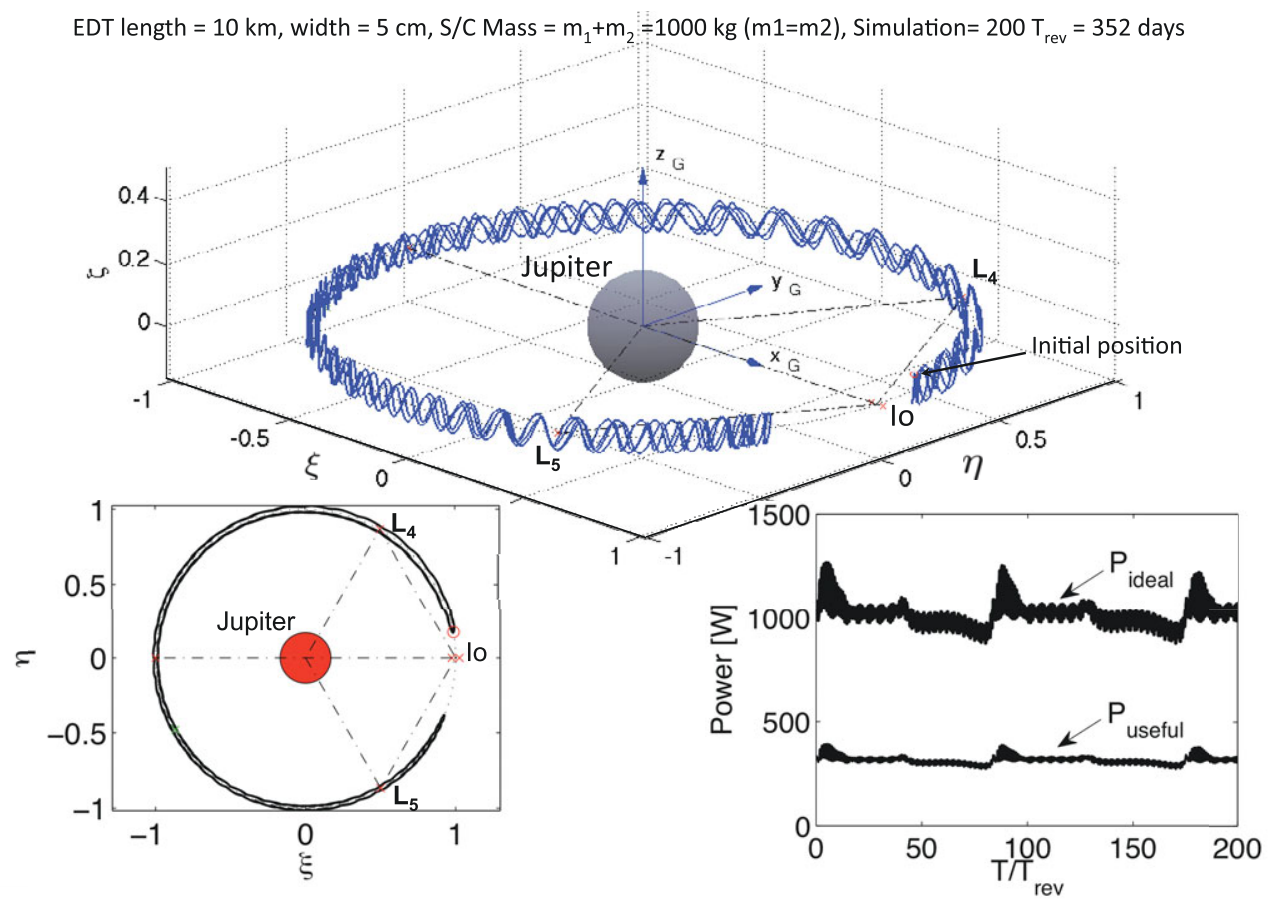

Figure 2. Horse-shoe orbit along the Io plasma torus

\subsection{Useful electrical power}

When the conductive wire is closed on an electrical load, the EDT generates useful onboard power. The power extraction occurs in conjunction with the electrodynamic force acting on the system, and it is generated at the expense of the energy of corotating plasma. Ideal power corresponds to zero load impedance and negligible ohmic losses, so useful power at the load is a fraction of $P_{\text {ideal }}$, typically $\eta \approx 0.3$.

\section{Moons Tour With Propellantless Maneuvers}

At Io's orbit the plasma density is relatively strong $\left(\approx 2 \times 10^{3} \mathrm{~cm}^{-3}\right)$ : electrodynamic interaction between the plasmasphere and tether can be exploited to change the orbital profile by means of propellanless maneuvers. Moreover, the Plasma Torus is above the stationary orbit of Jupiter, so two orbital maneuvers are possible with an EDT:

- orbit raising

- orbit lowering

An initial orbit with perijove $r_{p}$ at Io's distance and apojove $r_{a}$ at Europa's orbit is considered. For orbit raising, the electrodynamic force is exploited to increase the total energy of the dynamical system. In this case the satellite orbit is prograde (counter-clockwise) with the velocity vector $\vec{v}$ aligned in the same direction of $\vec{f}_{e l}$. At every perijove passage, where the plasma distribution is denser, the S/C energy increases. In this way, its possible to pump energy into the system by removing it from the plasmasphere. The greatest effect is on the apojove: in fact, while the $r_{p}$ remains more or less at the same altitude, $r_{a}$ tends to increase significantly.

For orbit lowering the satellite must be on a retrograde orbit and $\vec{v}$ is in the opposite direction of $\vec{f}_{e l}$. In this case a drag force brakes the S/C and the mechanical energy of 
the system decreases. At every perijove passage the orbit tends to change its eccentricity by lowering the apojove.

\section{Radiation Dose}

The magnetic field of Jupiter is very strong and its magnetosphere is the seat of intense radiation, that is seriously dangerous to the satellite. The Divine-Garretts model divides the charged particles in three main components as a function of their energetic level: the radiation belts include high energetic electrons and protons up to $1000 \mathrm{MeV}$. The GIRE (Galileo Interim Radiation Electron) model was used to quantify the fluences $\left[\right.$ particles $\left./ \mathrm{cm}^{2}\right]$ of highly energy electrons and protons, trapped in the radiation belts, on the satellite along the trajectory. As a result, the Io's Torus, situated at $5.5-10 R_{J}$, is a region of the magnetosphere with high density of charged particles, and if not appropriately shielded a satellite, orbiting inside the Io torus, will be exposed to high radiation doses: several hundreds MRad in a year mission.

\section{Conclusion}

The dynamics of a dumbell tether placed in the proximity of triangular Lagrangian points of the Jupiter-Io system was studied. New equilibrium positions were discoverd in the corotating frame. Their location depends on the system sizes (S/C mass, tether length and width). Moreover, the stability of these new points was investigated revealing that at high values of the Lorentz force only the superior equilibrium point remains stable and it moves more and more toward Io as the Lorentz force increases. The strong Jovian magnetic field, high electron density of the Plasma Torus and relative velocity of the satellite with respect to the plasmasphere make the EDT a convenient propellantless system for producing thrust (and consequently orbital maneuvres) and on board power. In fact, $\mathrm{kWs}$ of electrical power can be extracted from the Plasma Torus: about $1 \mathrm{~kW}$ of useful power with a rotating tether $20 \mathrm{~km}$ long. At the same time, by exploiting the electrodynamic interaction between an EDT and the plasma of Io's Torus it is possible to change the orbital parameters and make propellantless maneuvers to navigate among the Galilean Moons.

\section{References}

Bombardelli C., Lorenzini E. C., Curreli D., Sanjurjo-Rivo M., Lucas F. R., Peláez J. Scheeres D. J., \& Lara M. (2008), AIAA/AAS Astrodynamics Specialist Conference, Honolulu, Hawaii, USA

Curreli D., Lorenzini E. C., Bombardelli C., Sanjurjo-Rivo M., Lucas F. R., Peláez J., Scheeres D. J., \& Lara M. (2009), AAS 09-240, 19th AAS/AIAA Space Flight Mechanics Meeting, Savannah, Georgia, USA

Divine N. \& Garrett H. B. (1983), Journal of Geophysical Research, Vol. 88, No. A9, pp. 68896903.

Garrett H. B., Jun I., Ratliff J. M., Evans R. W., Clough G. A., \& McEntire R. W. (2003), National Aeronautics and Space Administration, JPL Publications 03-006

Peláez J., Sanjurjo-Rivo M., Lucas F. R., Lara M., Lorenzini E. C., Curreli D., \& Sheeres D. J. (2008), Final Report Ariadna Study 07/4201

Sanmartín J. R., Martínez-Sanchez M., \& Ahedo E. (1993), Journal of Propulsion and Power, Vol. 9, No.3, pp. 353-360 